\title{
Primary lung lymphoma involving the superior vena cava
}

\author{
Sen Wei, Xin Li, Xiaomin Qiu, Honglin Zhao, Gang Chen, Jun Chen ${ }^{*}$ and Qinghua Zhou*
}

\begin{abstract}
Primary lung lymphoma (PLL) presenting as a primary pulmonary lesion is rare and usually affects elderly people. Here we describe a 25-year-old Chinese man diagnosed with primary lung lymphoma, which presented as a huge lung tumor mimicking a primary lung cancer and involving the superior vena cava. He underwent double-sleeve reconstructions of bronchus and pulmonary arteries with right upper- and middle-lobe lobectomy along with replacement of the superior vena cava with a graft, and was then given standard chemotherapy of CHOP plus Rituximab. The patient has been well, showing no local recurrence or distal metastasis during a 27-month follow-up.
\end{abstract}

Keywords: Primary lung lymphoma, Lobectomy, Superior vena cava

\section{Background}

Primary lung lymphoma (PLL) is a rare distinct entity $(0.4 \%$ of all lymphomas) that usually affects elderly patients [1-3]. Generally, these cases are difficult to diagnose accurately because of a nonspecific clinical and radiological presentation. On the other hand, they have relatively satisfactory outcomes, especially in cases amenable to surgical resection. Owing to the rarity of PLL, standard treatment protocols have not yet been optimized, and there is no guideline as to when surgery is indicated.

Herein, we present a young patient with a rare primary pulmonary lymphoma (diffuse large B cell lymphoma, DLBCL) mimicking a primary lung cancer and involving the superior vena cava. He was treated by double-sleeve reconstructions of bronchus and pulmonary arteries with right upper and middle lobe lobectomy, and replacement of the superior vena cava with a graft.

\section{Case presentation}

A 25-year-old Chinese male was admitted because of respiratory symptoms and a large mass on the computed tomography $(\mathrm{CT})$ scan suggestive of primary lung carcinoma. This patient presented with 45 days of an irritating dry cough and right chest pain without other abnormal

\footnotetext{
* Correspondence: huntercj2004@yahoo.com; zhouqh1016@yahoo.com.cn Department of Lung Cancer Surgery, Tianjin Key Laboratory of Lung Cancer Metastasis and Tumor Microenvironment, Tianjin Medical University General Hospital, Tianjin 300052, China
}

findings such as fever, wheezing, hemoptysis, or superior vena cava syndrome. He had no smoking history and no family history of lung cancer. A review of systems was noncontributory. On admission, peripheral blood count, serum chemistry, and urinalysis were normal.

An enhanced chest CT scan revealed a huge pulmonary tumor over the right upper lung field with mediastinal and hilar lymphadenopathy surrounding the right upper lobe bronchus. Angiography showed encroachment on the superior vena cava (Figure 1). Abdominal CT, MRI scan of the brain, and a bone scan were all normal. Bronchoscopy showed a subsegmental bronchus of the right upper lobe being pressured from outside without mucosal invasion, and bronchoscopic biopsy did not show any malignancy. Before hospitalization, the patient had undergone a CT-guided fine-needle aspiration biopsy in another medical center and a preoperative diagnosis of squamous cell carcinoma was made. This conclusion later proved to be a misdiagnosis.

On 21 December, 2009, after establishment of a venous bypass between the right internal jugular vein and the right femoral vein, a surgical exploration was performed through a standard posterolateral incision under general anesthesia. There was a huge tumor $\left(18 \times 16 \times 15 \mathrm{~cm}^{3}\right)$ located in the right upper lobe surrounding the roots of the right upper lobe bronchus and invading the surrounding tissues and organs, including part of the right middle lobe, the middle and lower portion of the superior vena cava, the right side of the pericardium, the right 

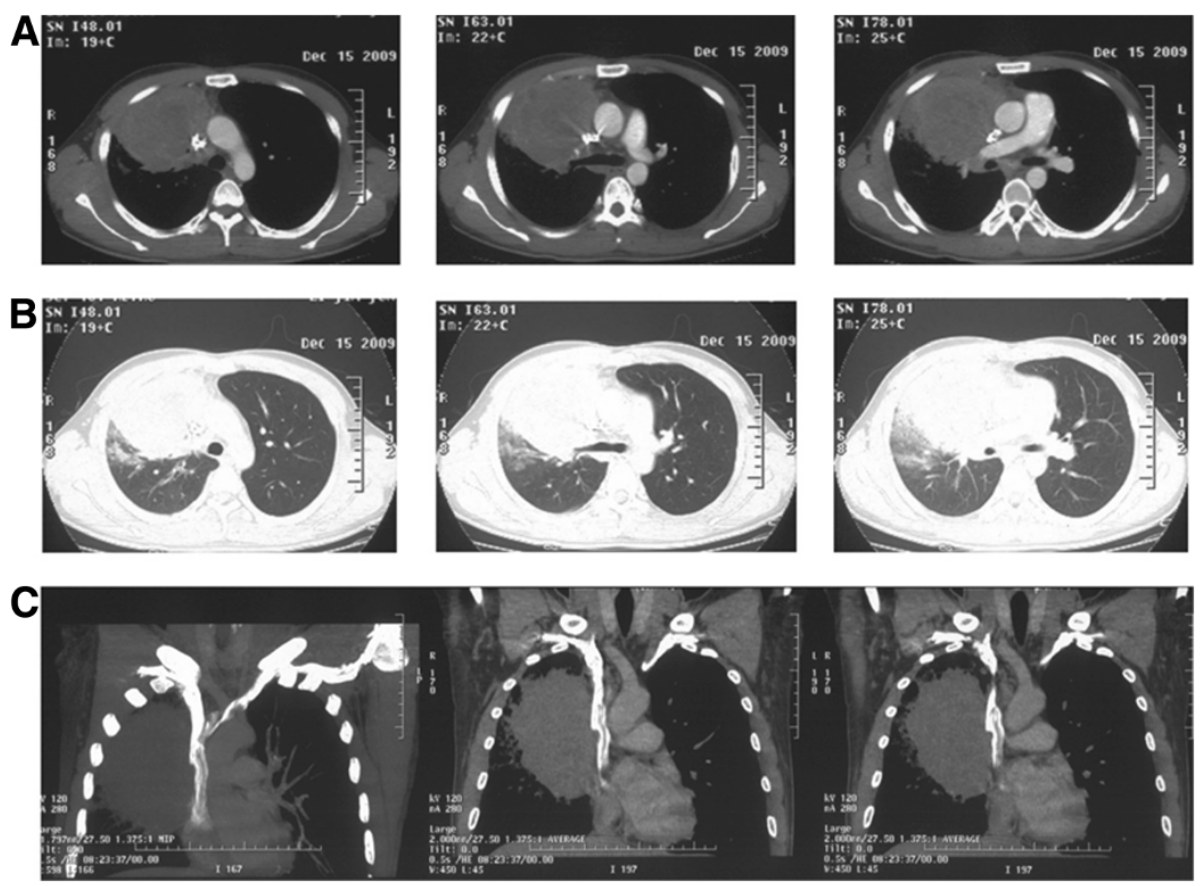

Figure 1 Enhanced chest CT scan before operation. (A) In the mediastinal window, the CT scan revealed the tumor encroaching on the superior vena cava (right panel), surrounding the right upper lobe bronchus (middle panel), and invading the right pulmonary artery (left panel). (B) In the lung window. (C) In the mediastinal window, coronal.

phrenic nerve, and the trunk of right pulmonary artery (Figure 2A). Several oval nodules found at the same time in the middle lobe were suspected to be metastatic lesions. Mediastinal and hilar lymph nodes were enlarged and had a tendency to integration. Intraoperative frozen section of lymph node sampling proved all of them to be metastatic poorly differentiated carcinoma.

Based on these findings, sleeve lobectomy of the right upper and middle lobes was performed successfully to achieve complete tumor resection. Portions of the surgical procedure were sophisticated, including sleeve resection and reconstruction of the right bronchus and pulmonary artery, reconstruction of the superior vena cava, partial resection of the pericardium, and systematic mediastinal lymphadenectomy (Figure 2B). Postoperative histopathological assessment of the huge lobulated grayyellow lung mass revealed that the tumor was situated in the lung parenchyma and involved visceral pleura. The tumor cells exhibited large vesicular nuclei and frequent mitoses without tumor necrosis. Immunohistochemically, the tumor cells expressed CD20, CD23, CD30, CD43, Kappa, and MUM1, but not CD117, TdT, CD10, or Bcl-6. The proliferation fraction as determined by staining with Ki-67 was $80 \%$. Based on these findings, the diagnosis of diffuse large B cell lymphoma (Figure 3) was made. The diagnosis of PPL was based on characteristic histological and immunophenotypical features according to the Kiel classification and the WHO classification [4]. Mediastinal

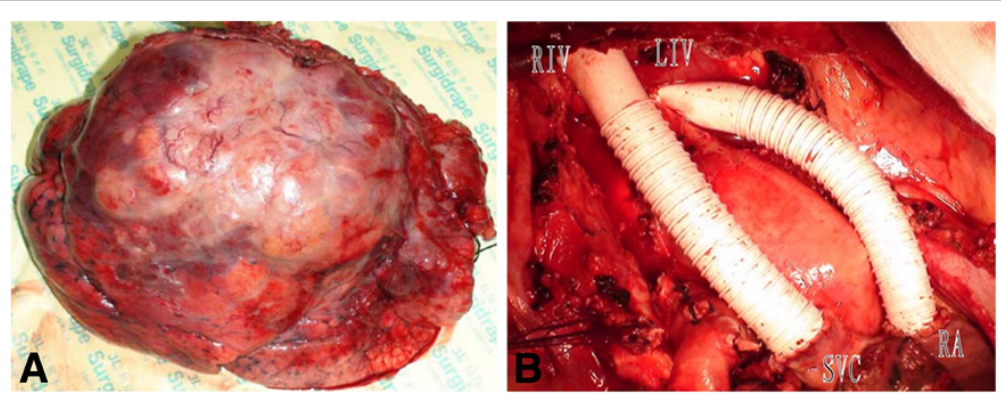

Figure 2 The images during the operation. (A) Gross features of the 15-cm mass in right upper-middle lobes. (B) SVC grafts: the graft on the left is from the right innominate vein (RIV) to the superior vena cava (SVC); the graft on the right is from the left innominate vein (LIV) to the right atrium (RA) 

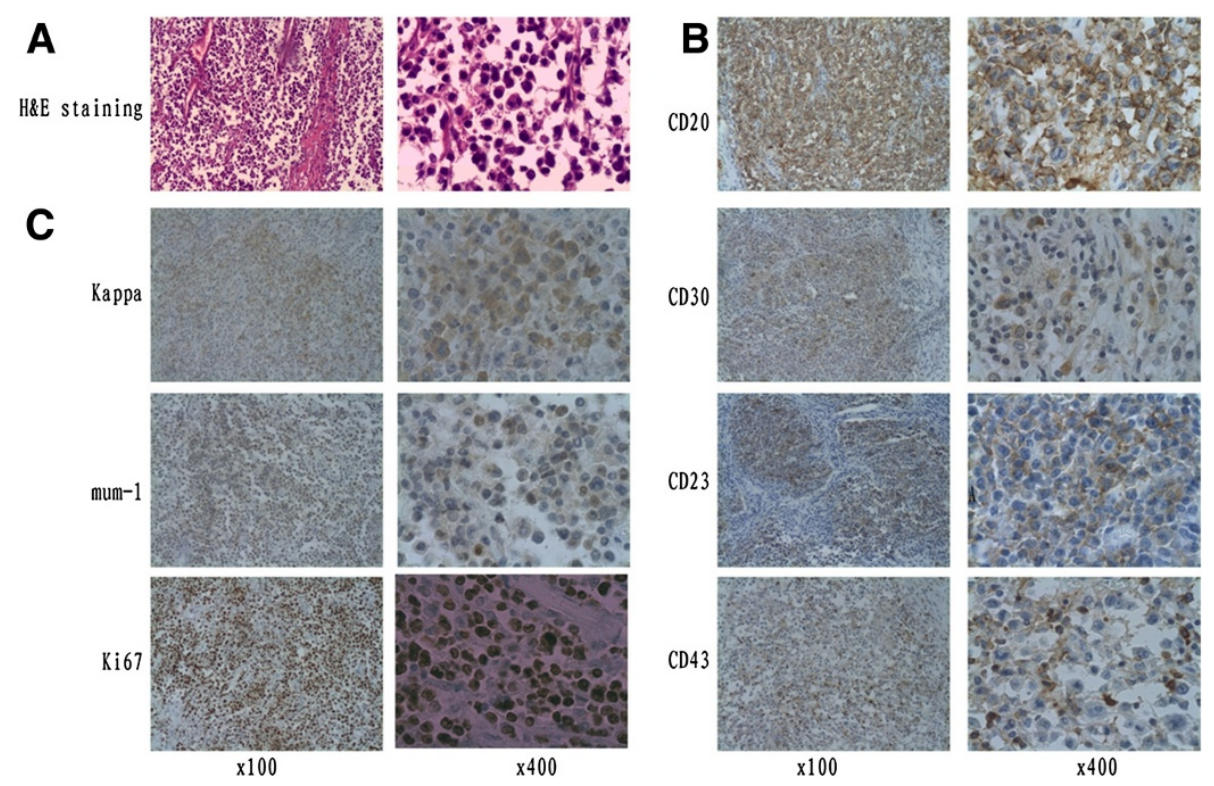

Figure 3 Histopathological images (×100 and $\times 400)$. (A) Hematoxylin and eosin (H\&E) staining of primary lung lymphoma. (B)

Immunohistochemical staining of primary tumor with antibodies to CD20, CD30, CD23, and CD43. (C) Immunohistochemical staining of primary tumor with antibodies to Kappa, mum-1, and Ki67.

[nos. \#2, 3, 4, 7 and 9] and hilar [nos. \#10] lymph nodes contained tumor. All of the negative surgical margins (bronchus, pulmonary artery, and superior vena cava) were confirmed. The tumor was determined to be stage IIE by the Ann Arbor classification system [5].

Five weeks after the operation, the patient received adjuvant therapy with CHOP (cyclophosphamide, Adriamycin, vincristine and prednisone) and Rituximab (a humanized monoclonal anti CD-20 antibody) for six cycles. For economic reasons, he did not receive maintenance therapy of Rituximab. After a 27-month follow-up period, as shown in Figure 4 for16-month after operation, the patient is well and without evidence of locally recurrent or distal disease.

\section{Discussion}

Extranodal lymphomas account for $24 \%$ to $48 \%$ of NHL, and primary pulmonary lymphomas are infrequent (about 3.6\% among all extranodal lymphomas) [2]. Primary pulmonary lymphoma (PPL) is defined as a clonal lymphoid proliferation affecting one or both lungs (parenchyma and/or bronchi) in a patient with no detectable extrapulmonary involvement at diagnosis or during the subsequent 3 months [6,7]. According to the World Health Organization's classification system, the most common histological subtypes of primary pulmonary lymphoma are low-grade lymphomas (75-88\%), and the second most frequent histological type of lymphoma to involve the lung is diffuse large B-cell lymphoma (DLBCL), which represents only about $5-20 \%$ of primary pulmonary lymphomas [8]. The high-grade B-cell PPLs usually spread rapidly into mediastinal and extrathoracic locations. The case of DLBCL we reported here presented as a huge lung tumor mimicking a primary lung cancer involving the superior vena cava.

Primary pulmonary lymphoma is most commonly seen in between the 5th and 7th decades of life, with a mean age of 50 years and a male-to-female ratio of 1.07:1 [9]. The present case was a young (25-year-old) man with nonspecific symptoms of irritating dry cough and right chest pain. As reported previously, PPLs can present with nonspecific symptoms [10]. Since PPLs can present with different radiological manifestations, an accurate diagnosis is often not easy. In our case, the images were suggestive of locally advanced lung cancer, but the final diagnosis turned out to be a primary pulmonary lymphoma. This further emphasizes the importance of histological diagnosis of lung lesions, although this tumor was even misdiagnosed as lung cancer before operation based on pathological findings from CT-guided fine-needle aspiration biopsy. In the literature, preoperative CT-guided fine-needle aspiration biopsy allows diagnosis of PPL in only $25 \%$ of patients [11]. Thus, in order to avoid the mistake of the misdiagnosis of this curable disease, it is important to keep in mind that any radiological abnormality of the lung parenchyma may be a lymphoproliferative disorder, which sometimes has a good prognosis and has a very different management plan from epithelial neoplasia of the lung, especially when the lung is primarily affected [12]. 

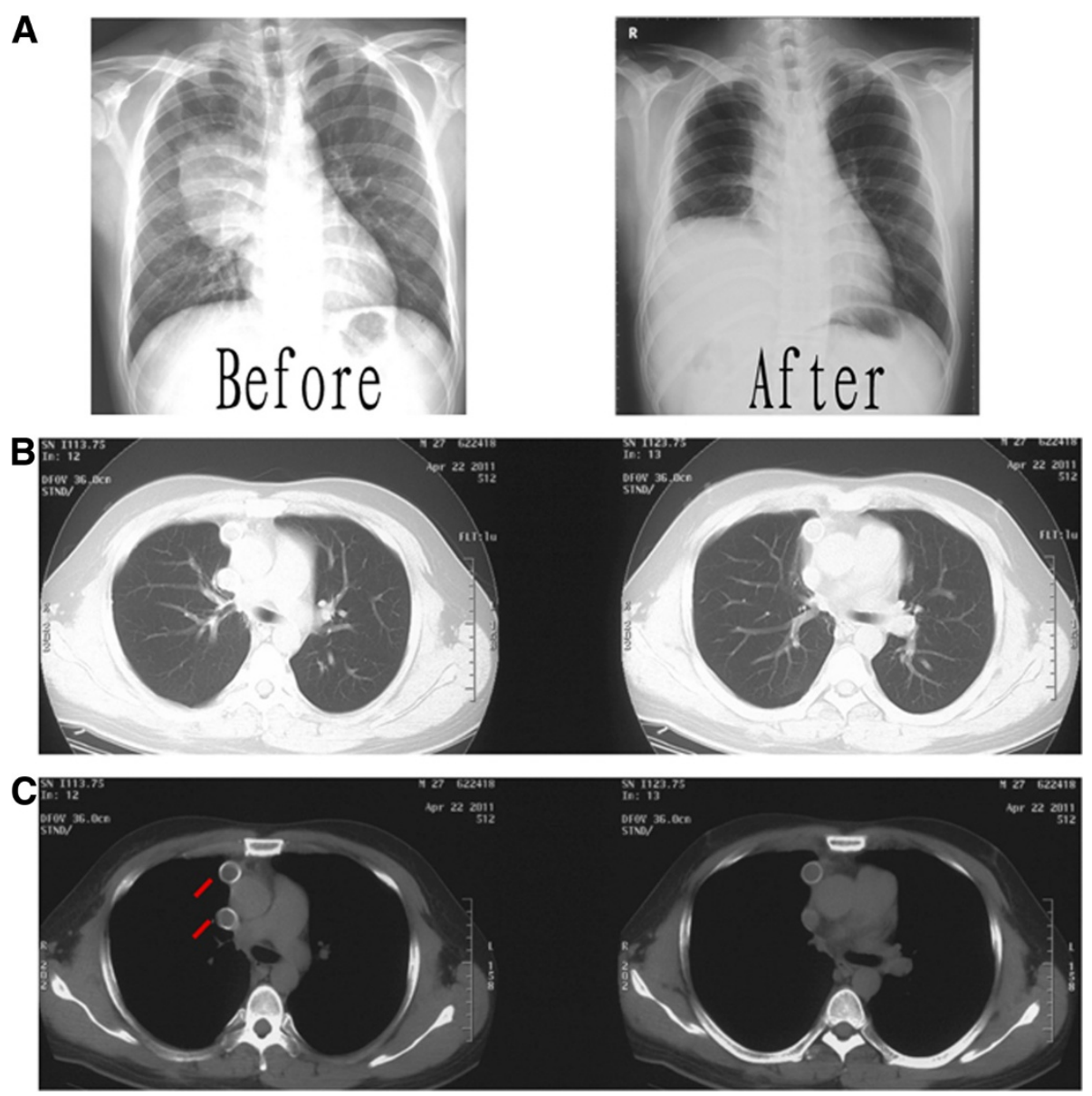

Figure $\mathbf{4}$ Chest X-ray and CT scan after operation. (A) The chest x-ray images of the patient before/after operation. (B and C) The chest CT scan 16 months after the operation. The red lines show the SVC replacement graft.

High-grade pulmonary B-cell lymphoma is far rare and usually occurs in individuals with an underlying disorder (e.g., immunodeficiency). The prognosis is poor compared with low-grade lymphoma, and therapeutic options depend on the underlying disorder. Because of its rarity, relatively little is known about the biological characteristics of DLBCL. Treatment of these rare tumors is poorly standardized. Several treatment options are available, including tumor resection, surgery with adjuvant chemotherapy, or chemotherapy alone. The optimal treatment remains controversial, but the prognosis can be relatively excellent [13]. One large series of 70 patients reported a $94 \%$ survival at 5 years for low-grade primary pulmonary lymphoma, and a median survival of 3 years for high-grade disease [10]. Another series with 48 patients reported a 68\% 5-year survival for primary pulmonary MALT lymphoma and a 65\% 5-year survival for non-MALT lymphoma [3].

We believe surgery should be the treatment of choice if a complete resection can be achieved. The potential surgical candidate could be any patient with locally resectable tumor up to stage IIE. Lymph node involvement does not appear to be a contraindication to surgery [14]. To our knowledge, few patients with pulmonary DLBCL involving the superior vena cava have received radical surgical treatment, so this is an extremely uncommon case. Although some authors have recommended a pneumonectomy for multiple lesions of lowgrade PPL involving one lung, in our view this option may be too aggressive because of the indolent course of the disease. One patient reported by Frederic et al. [15] is still alive after a 36-month follow-up.

Treatment after surgical resection is often based on a combination chemotherapy regimen. Diffuse large B cell lymphoma is frequently treated by CHOP (cyclophosphamide, Adriamycin, vincristine and prednisone), and Rituximab may improve the response to CHOP treatment, as has been shown in systemic diffuse large B-cell lymphoma. Although survival is worse for patients with highgrade PPL than for those with low-grade PPL, published reports show that more than half of patients with highgrade PPL can achieve survivals of 8 to 10 years [16]. 


\section{Conclusion}

In conclusion, surgery should be the treatment of choice in cases of localized PPL when complete resection can be achieved.

\section{Consent}

Written informed consent was obtained from the patient for publication of this Case report and any accompanying images.

\section{Competing interests}

The authors declare that they have no competing interests.

\section{Authors' contribution}

SW performed research, analyzed data, and wrote the paper; $X L, H L Z$, XMQ and GC collected the data and followed up the patient; JC performed operation and research, analyzed data, and wrote the paper; $\mathrm{QHZ}$ performed operation and revised the manuscript. All authors read and approved the final manuscript.

\section{Acknowledgements}

This study was partly supported by grants from the Science and Technology Support Key Program of Tianjin (09ZCZDSF04100, 09ZCZDSF04000) and the project of Ministry of Education for New Century Excellent Talents (NCET-10-0956).

Received: 8 February 2012 Accepted: 3 June 2012

Published: 30 June 2012

\section{References}

1. Rosenberg SA, Diamond HD, Jaslowitz B, Craver LF: Lymphosarcoma: A review of 1269 cases. Medicine 1961, 40:31-84.

2. Freeman C, Berg JW, Cutler SJ: Occurrence and prognosis of extranodal lymphomas. Cancer 1972, 29:252-260.

3. Ferraro P, Trastek VF, Adlakha H, Deschamps C, Allen Ms, Pairolero PC: Primary non-Hodgkin's lymphoma of the lung. Ann Thorac Surg 2000, 69:993-997.

4. Harris NL, Jaffe ES, Stein $H$, et al: A revised European-American classification of lymphoid neoplasms: a proposal from the International Lymphoma Study Group. Blood 1994, 84:1361-1392.

5. Carbone PP, Kaplan HS, Musshoff K, Smithers DW, Tubiana M: Report of the Committee on Hodgkin's Disease Staging Classification. Cancer Res 1971, 31:1860-1861.

6. Cadranel JWM, Antoine M: Primary Pulmonary Lymphoma. Eur Respir J 2002, 20:750-762.

7. Kim JH, Lee SH, Park J, Kim HY, Lee SI, Park JO, Kim K, Kim WS, Jung CW, Park YS, Im YH, Kang WK, Lee MH, Park K, Han JH, Ko YH: Primary pulmonary non-Hodgkin's lymphoma. Jpn J Clin Oncol 2004, 34:510-514.

8. Nicholson AG, Harris NL: Primary pulmonary diffuse large B-cell lymphoma. In World Health Organization classification of tumours. Pathology and genetics. Tumours of the lung, pleura, thymus and heart. Edited by Travis WD, Brambilla E, Muller-Hermelink HK, Harris CC. Lyon: IARC Press; 2004:91.

9. Li G, Hansmann ML, Zwingers T, Lennert K: Primary pulmonary lymphoma of the lung: Morphological, immunohistochemical and clinical features. Histopathology 1990, 16:519-531.

10. Cordier JF, Chailleux E, Lauque D, et al: Primary pulmonary lymphomas: a clinical study of 70 cases in nonimmunocompromised patients. Chest 1993, 103:201-208.

11. Pisani RJ, DeRemee RA: Clinical implications of the histopathologic diagnosis of pulmonary lymphomatoid granulomatosis. Mayo Clin Proc 1990, 65:151-163.

12. Hu RL, Li H: Giant cystic hemolymphangioma of the postmediastinum. Thoracic Cancer 2010, 1:175-177.

13. Mattedi RL, Bernardi Fdel C, Bacchi CE, Siqueira SA, Mauad T: Fatal outcome in bronchus-associated lymphoid tissue lymphoma. J Bras Pneumo/ 2007, 33:487-491.

14. Eckardt J, Olsen KE, Petersen H: Metastasis in the subcarinal lymph node with unknown primary tumor. Thoracic Cancer 2011, 2:69-70.
15. Vanden Eynden F, Fadel E, de Perrot M, de Montpreville V, Mussot S, Dartevelle P: Role of surgery in the treatment of primary pulmonary B-cell lymphoma. Ann Thorac Surg 2007, 83:236-240.

16. L'Hoste RJ Jr, Filippa DA, Lieberman PH, Bretsky S: Primary pulmonary lymphomas. A clinical pathologic analysis of 36 cases. Cancer 1984, 54:1397-1406.

doi:10.1186/1477-7819-10-131

Cite this article as: Wei et al:: Primary lung lymphoma involving the superior vena cava. World Journal of Surgical Oncology 2012 10:131.

\section{Submit your next manuscript to BioMed Central and take full advantage of:}

- Convenient online submission

- Thorough peer review

- No space constraints or color figure charges

- Immediate publication on acceptance

- Inclusion in PubMed, CAS, Scopus and Google Scholar

- Research which is freely available for redistribution 